African Crop Science Journal by African Crop Science Society is licensed under a Creative Commons Attribution 3.0 Uganda License. Based on a work at www.ajol.info/ and www.bioline.org.br/cs DOI: https://dx.doi.org/10.4314/acsj.v27i3.1

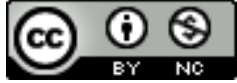

\title{
ELÉMENTS DE PRÉCISION DES AIRES DE DISTRIBUTION DU FONIO ET DE SES APPARENTÉS SAUVAGES EN FONCTION DES ZONES CLIMATIQUES, DES TYPES DE VÉGÉTATION ET DE SOLS AU SÉNÉGAL
}

\author{
A. NGOM, M.S. MBAYE, M. GUEYE, C. BASSENE ${ }^{1}$, A.A. CAMARA et K. NOBA \\ Laboratoire de Botanique et Biodiversité, Département de Biologie Végétale, Université Cheikh \\ Anta Diop, BP 5005 Dakar-Fann, Sénégal \\ ${ }^{1}$ UFR Sciences Agronomiques, Aquaculture et Technologies Alimentaires, Université Gaston Berger \\ de Saint-Louis, BP 234 Saint-Louis, Sénégal \\ Auteur de correspondance : ablaye11.ngom@ucad.edu.sn
}

(Received 2 October 2018; accepted 31 July 2019)

\section{RESUME}

Le fonio blanc (Digitaria exilis Stapf) est l'une des céréales les plus prometteurs en Afrique de l'Ouest grâce à ses qualités nutritionnelles et de génération de revenus par les femmes. Au Sénégal, sa culture a régressé ces dernières années de même que l'aire de répartition de ses parents sauvages. Cette étude vise ainsi à préciser l'écologie des apparentées sauvages du fonio. Les données utilisées proviennent de collections des Herbiers DAKAR et IFAN, de la base de données GBIF et de collectes de terrain qui ont permis de dessiner des cartes de répartition des espèces en fonction des zones climatiques, des types de végétation et de sols. Les résultats de l'étude ont révélé que la zone soudanienne est la plus riche en termes de diversité spécifique avec 11 espèces inventoriées. Les espèces occupent tous les types de végétation du Sénégal et la présence de certaines en zones arides, de type steppe, et humides composées principalement de forêts et de savanes boisées témoigne leur remarquable adaptation à des conditions environnementales contrastées. Il ressort également de cette étude que les espèces affectionnent mieux les sols ferrugineux tropicaux, les lithosols, les sols ferralitiques et hydromorphes. Digitaria ciliaris et D. gayana se rencontrent sur presque l'ensemble des types de sols. Quant à $D$. exilis, elle pousse sur les mêmes sols que $D$. longiflora, son plus proche parent sauvage. Le développement de banques de semences assurant la conservation ex situ de ces espèces sauvages, sources potentielles de gènes pour le fonio, serait d'une importance primordiale pour la sauvegarde de cette biodiversité.

Mots Clés : Conservation, écologie, fonio, Sénégal, parents sauvages

\begin{abstract}
White fonio (Digitaria exilis Stapf) is one of the most promising cereals in West Africa due to its nutritional qualities and for its potential to generate financial returns for women. In Senegal, its culture has declined in recent years as well as the distribution area of its wild relatives. This study aims to
\end{abstract}


specify the ecology of wild relatives of fonio. The data used come from collections of DAKAR and IFAN Herbaria, the GBIF database and field collections which made it possible to draw species distribution maps according to the climatic zones, vegetation and soils types. The results of the study revealed that the Sudanian zone is the richest in term of specific diversity with 11 inventoried species. The species occupy all types of vegetation in Senegal and the presence of some in arid zones, steppe type, and wet zones composed mainly of forests and wooded savannahs attests their remarkable adaptation to contrasting environmental conditions. It is also apparent from this study that species prefer tropical ferruginous soils, lithosols, ferralitic and hydromorphic soils. Digitaria ciliaris and $D$. gayana occur on almost all types of soils. As for D. exilis, it grows on the same soil as D. longiflora, its closest wild relative. The development of seed banks for the ex situ conservation of these wild species, potential sources of genes for fonio, would be of primary importance for the protection of this biodiversity.

Key Words: Conservation, ecology, fonio, Senegal, wild relatives

\section{INTRODUCTION}

Le fonio blanc (Digitaria exilis Stapf) est connu pour son importance dans l'alimentation humaine du fait de ses qualités nutritionnelles et thérapeutiques qui font de cette céréale une source nutritionnelle stratégique (Asfaw, 2006 ; Adoukonou-Sagbadja et al., 2007 ; Popkin et al., 2012 ; Diop et al., 2018). Ces propriétés permettent de générer des rendements financiers importants pour les agriculteurs et, de manière significative, pour les femmes, qui sont traditionnellement impliquées dans la transformation et la commercialisation (USAID, 2008 ; Paraïso et al., 2011 ; Diop et $a l ., 2018)$. Il contribue ainsi à la sécurité alimentaire, une préoccupation croissante pour les pays de la région subsaharienne.

Au Sénégal, le fonio est principalement cultiveì dans sa partie sud où il constitue un aliment de base complémentaire pour les populations des zones rurales (Vall et al., 2011 ; Gueye et al., 2015). Cependant, au cours des dernières années, une disparition progressive de la couverture terrestre dus aux impacts négatifs liés aux pressions anthropiques et des conditions climatiques précaires a entrainé un rétrécissement considérablement de l'aire de distribution de cette plante cultivée (Ministère de l'Agriculture, 1996 ; Diop et al., 2018) ainsi que celle de ses parents sauvages. Face à cette perte de diversité, la conservation de ces espèces sauvages demeure une étape importante pour leur utilisation qui se heurte toutefois al de nombreux obstacles dont celui d'une connaissance insuffisante de leur aire de distribution.

En effet, au Sénégal, les études sur la répartition des digitaires sont obsolètes (Berhaut, 1967 ; Vanden Berghen, 1991) et certaines espèces telles que Digitaria aristulata, Digitaria gentilis et D. patagiata sont devenues respectivement rares, endémiques ou menacées de disparition (USAID, 2008). Par conséquent, la collecte de nouvelles données pourrait être cruciale pour déterminer la répartition géographique actuelle des espèces sauvages, source de gènes utilisables dans l'amélioration de l'espèce cultivée, en fonction de facteurs environnementaux.

Cet article vise à fournir une mise à jour des connaissances sur la répartition géographique et l'écologie des parents sauvages du fonio en fonction des zones climatiques, des types de végétation et de sols du Sénégal.

\section{METHODOLOGIE}

Les espèces étudiées sont $D$. argillacea, $D$. aristulata, D. ciliaris, D. debilis, D. delicatulata, $D$. diagonalis, $D$. exilis, $D$. gayana, D. horizontalis, D. leptorhachis, $D$. longiflora, D. nuda, D. patagiata, D. perrottetii, D. ternata et $D$. velutina. Les 
coordonnées géographiques des collections d'herbiers de toutes les espèces étudiées issues des Herbiers DAKAR et IFAN (Université Cheikh Anta Diop) ont été enregistrées. Ces données ont été complétées par celles de la base de données GBIF (Global Biodiversity Information Facility) et celles de notre collection (spécimens de D. ciliaris, D. exilis, $D$. horizontalis, $D$. longiflora et $D$. perrottetii collectés dans les régions de Dakar, Thiès et Ziguinchor). Dans cette étude, trois facteurs écologiques ont été retenus : le climat, la végétation et le sol. La carte des zones climatiques du Sénégal utilisée est définie par Mbow (2009) alors que les caractéristiques des types de végétation et de sol ont été fournies par le Centre de Suivi Ecologique (2007).
Une approche de géoréférencement basée sur la sélection de cartes avec le logiciel QGIS (version 2.18.14, 2015) a été utilisée pour analyser la distribution des espèces. Une telle approche a permis d' attribuer des coordonnées géographiques à ces cartes qui sont en fichier image. La projection des coordonnées géographiques des différents individus sur les cartes géoréférencées a permis d'obtenir l'aire de répartition des espèces.

\section{RESULTATS ET DISCUSSION}

Distribution géographique en fonction des zones climatiques du Sénégal. La Figure 1 montre la distribution des espèces en fonction des zones climatiques du Sénégal.

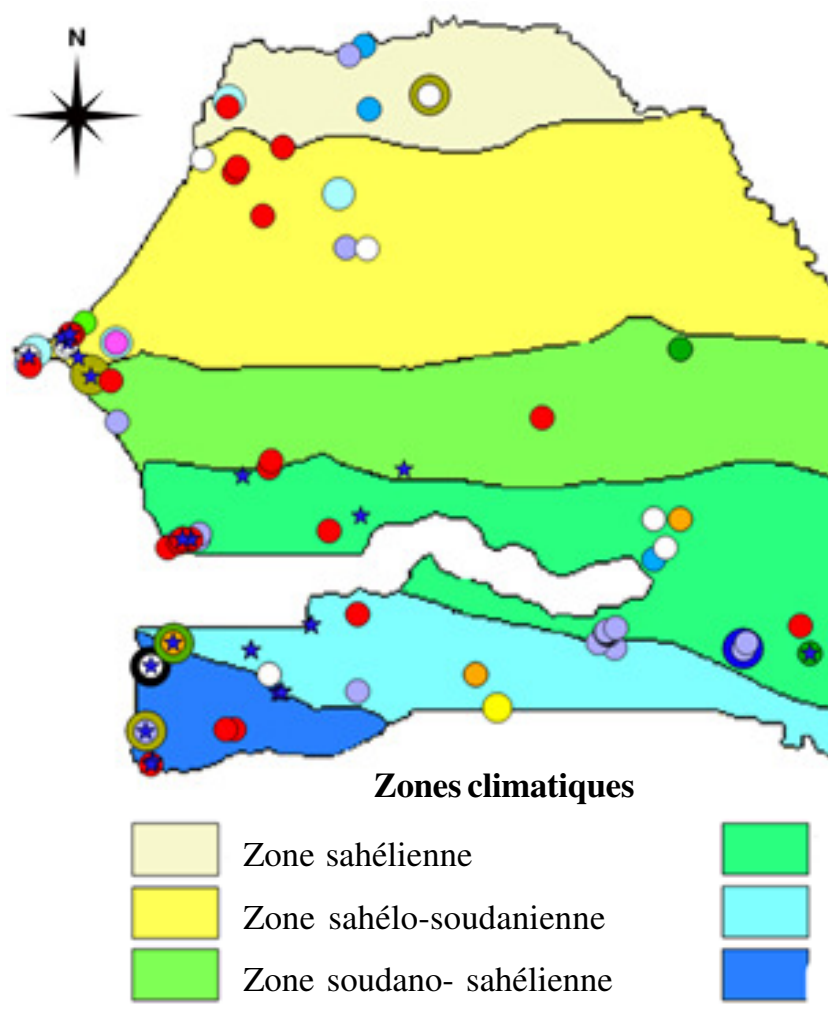

Zone soudano- sahélienne
Zone soudanienne

Zone soudano-guinéenne

Zone guinéenne

(C) QGIS 2015

Figure 1. Aires de répartition du fonio et de ses parents sauvages en fonction des zones climatiques du Sénégal. 
En termes de richesse en espèces, la zone soudanienne est la mieux représentée. Dans cette seule région, 11 espèces ont été inventoriées à ce jour, à savoir $D$. argillacea, $D$. aristulata, $D$. ciliaris, $D$. diagonalis, $D$. gayana, D. nuda, D. delicatula, D. exilis, D. horizontalis, D. longiflora et D. ternata. Au contraire, la zone soudano-sahélienne tend à être la zone géographique où la richesse spécifique est la plus faible avec quatre espèces inventoriées à ce jour à savoir $D$. aristulata, $D$. ciliaris, D. longiflora et $D$. velutina.

Dans l'ensemble, la richesse spécifique est indépendante du gradient climatique Nord-Sud. Toutefois, il est important de noter que 14 des 16 espèces étudiées sont rencontrées dans la partie sud du pays (la zone guinéenne, la zone soudano-guinéenne et la zone soudanienne). Cette partie sud constitue probablement la zone géographique et écologique ouİ la plupart des espèces de Digitaria y trouvent les conditions environnementales favorables à leur croissance.

De plus, bien qu'aucune des six zones climatiques ne soit individualisée par une flore particulière d'espèces, il est remarquable de noter que certaines espèces ont une aire de répartition étendue (Fig. 2). C'est le cas de $D$. ciliaris, $D$. horizontalis et $D$. longiflora qui couvrent toutes les zones climatiques du Sénégal. En effet, en ce qui concerne $D$. longiflora, des données supplémentaires provenant d'échantillons non géoréférencés ont confirmé sa présence dans la zone sahélienne, en particulier dans la région du fleuve Sénégal (Berhaut 5420).

À l'inverse, d'autres espèces ont une distribution plutôt étroite dans le pays. C'est le cas de $D$. diagonalis, $D$. ternata qui sont d'affinité soudanienne et $D$. patagiata, d'affinité guinéenne. Il s'agit également de $D$. argillacea, $D$. exilis et $D$. delicatula qui semblent être des espèces soudano-guinéennes. $D$. perrottetii, serait une espèce d'affinité sahélienne puisque rencontrée uniquement en zones sahélienne et sahélo-soudanienne tandis que $D$. aristulata a tendance à être d'affinité sahélo-soudanienne.

Enfin, nombre d'espèces sont rencontrées aussi bien au nord et au sud du pays. Bien qu'elles ne soient signalées dans toutes les zones climatiques, leur présence dans des zones

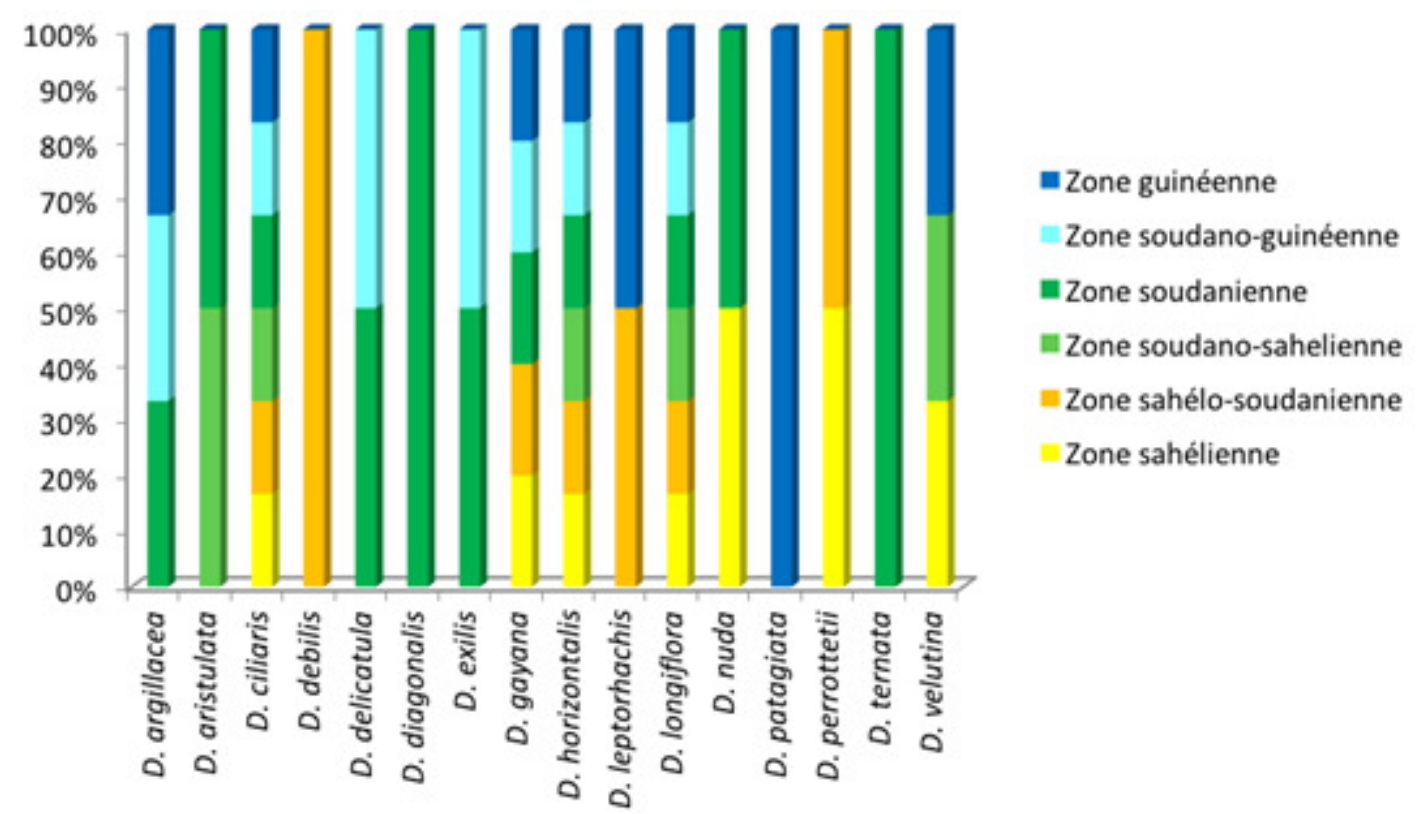

Figure 2. Zones climatiques couvertes par chacune des espèces rencontrées au Sénégal. 
contrastées (nord et sud) atteste l'absence d'affinité al̀ l'égard du type de climat considéré. Il s'agit de D. debilis, D. gayana, D. leptorhachis, D. nuda et D. velutina. S'agissant de $D$. debilis, les résultats de l'étude montrent qu'elle n'est présente qu'en zone sahélo-soudanienne. Pourtant, sa zone de couverture semble être plus importante puisqu'elle a été retrouvée au Sine-Saloum, en Basse Casamance (Vanden Berghen, 1991) et aÌ ThieÌs (Berhaut 2784).

Distribution géographique en fonction des types de végétation du Sénégal. La majeure partie de la végétation du Sénégal est constituée de savanes et de steppes (Fig. 3). A l'image des zones climatiques, les apparentés sauvages du fonio occupent tous les types de végétation du Sénégal. Si, dans leur intégralité, ces espèces supportent un large éventail de conditions environnementales, il convient néanmoins de mentionner que certaines d'entre-elles ont des caractéristiques remarquables. Qu'elles soient présentes dans toutes les zones climatiques ou non, ces apparentés sauvages se rencontrent à la fois en zones arides où la végétation est de type steppe (au nord) et en zones humides composées principalement de forêts et de savanes boisées (sud). C'est le cas de $D$.

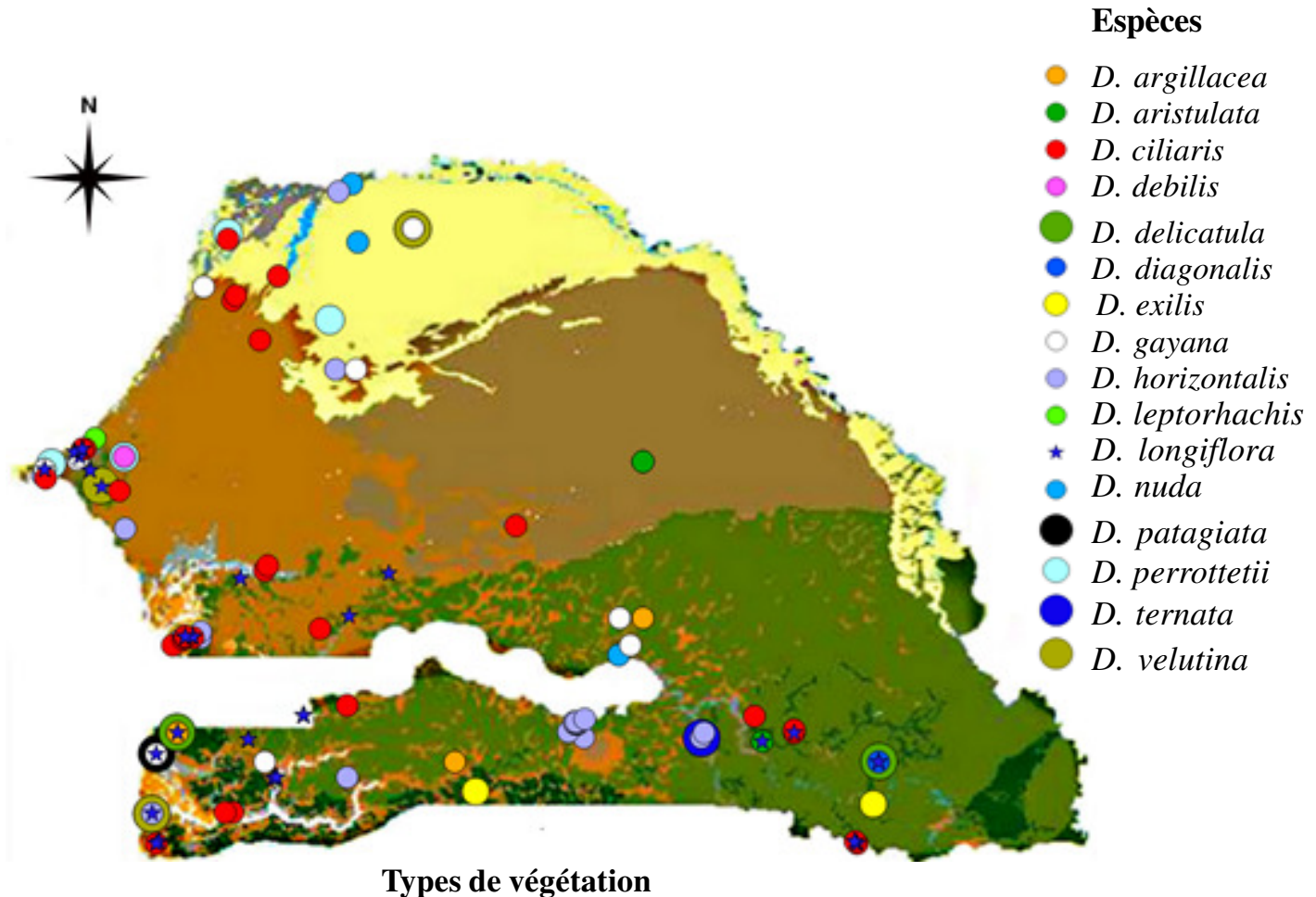

Types de végétation

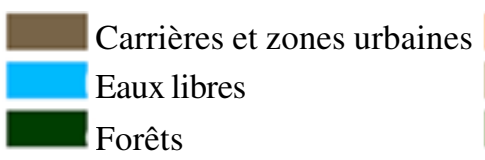

(C) QGIS 2015

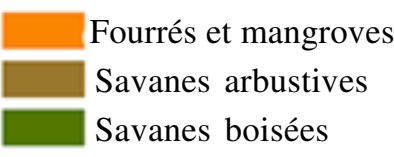

0
Steppes

Tannes et vasières denudées Zones de culture

Figure 3. Aires de répartition du fonio et de ses parents sauvages en fonction des types de végétation du Sénégal. 
ciliaris, D. gayana, D. horizontalis, D. (ii) à l'ouest : cas de D. debilis (zones de longiflora, D. nuda et D. velutina. culture).

Pour d'autres espèces, la particularité réside dans leur confinement :

(i) au sud : cas de D. delicatula (forêts et savanes boisées), D. diagonalis, $D$. ternata (savanes boisées), et $D$. patagiata (fourrés et mangroves). Il s'agit également de l'espèce cultivée, $D$. exilis, dont on trouve dans les savanes boisées à l'état sauvage.

Distribution géographique en fonction des types de sols du Sénégal. Les résultats de la présente étude montrent que les espèces étudiées, prises dans leur intégralité, poussent sur la plupart des sols du pays (Fig. 4). En effet, on les trouve sur des :

(i) sols bruns subarides et rouge- brun à fertilité relativement faible: C'est le cas de D. ciliaris, D. gayana, $D$.

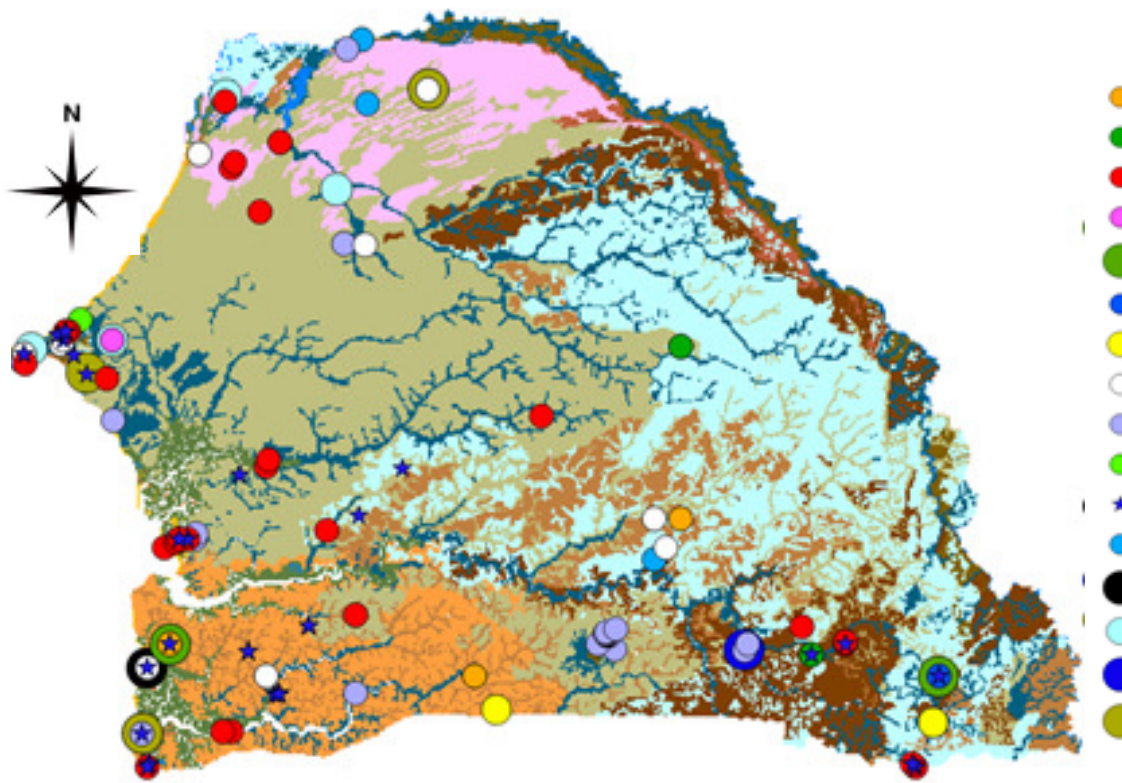

\section{Espèces}

- D. argillacea

- D. aristulata

- D. ciliaris

- D. debilis

D. delicatula

- D. diagonalis

D. exilis

D. gayana

- D. horizontalis

- D. leptorhachis

$\star$ D. longiflora

- D. nuda

D. patagiata

D. perrottetii

D. ternata

Types de sols

\begin{tabular}{|l|l|l|}
\hline Brun subarides & Ferrugineux tropicaus & Regosols \\
\hline Dunes littorales & Halomorphes & Rouge-brun \\
\hline Eau & Hydromorphes & Vasières \\
\hline Ferralitiques & Lithosols & Vertiques \\
& Peu évolués & $300 \mathrm{~km}$ \\
\hline C QGIS 2015 & 0 & V \\
\hline
\end{tabular}

Figure 4. Aires de répartition du fonio et de ses parents sauvages en fonction des types de sols du Sénégal. 
TABLEAU 1. Types de sols couverts par le fonio et ses parents sauvages au Sénégal

\begin{tabular}{|c|c|c|c|c|c|c|c|c|}
\hline \multirow[t]{2}{*}{ Espèces } & \multicolumn{8}{|c|}{ Types de sols } \\
\hline & $\begin{array}{l}\text { Sols bruns } \\
\text { subarides et } \\
\text { rouge-brun }\end{array}$ & $\begin{array}{l}\text { Sols } \\
\text { ferralitiques }\end{array}$ & $\begin{array}{l}\text { Sols ferrugineux } \\
\text { tropicaux }\end{array}$ & $\begin{array}{l}\text { Sols } \\
\text { hydromorphes }\end{array}$ & Lithosols & $\begin{array}{l}\text { Sols peu } \\
\text { évolués }\end{array}$ & Regosols & Vasières \\
\hline D. argillacea & - & + & - & - & + & - & - & - \\
\hline D. aristulata & - & - & + & - & + & - & + & - \\
\hline D. ciliaris & + & + & + & + & + & - & + & + \\
\hline D. debilis & - & - & + & - & - & - & - & - \\
\hline D. delicatulata & - & - & + & - & + & - & - & - \\
\hline D. diagonalis & - & - & - & - & + & - & - & - \\
\hline D. exilis & - & + & - & - & + & - & - & + \\
\hline D. gayana & + & + & + & + & + & + & - & + \\
\hline D. horizontalis & + & + & + & + & - & - & + & + \\
\hline D. leptorhachis & - & - & - & + & - & - & - & + \\
\hline D. longiflora & - & + & + & + & + & - & + & + \\
\hline D. nuda & + & - & + & + & - & - & - & - \\
\hline D. patagiata & - & + & - & - & - & - & - & - \\
\hline D. perrottetii & + & - & + & + & - & - & - & - \\
\hline D. ternata & - & - & - & + & - & - & - & - \\
\hline D. velutina & + & - & - & - & + & - & - & + \\
\hline
\end{tabular}


horizontalis, D. nuda, D. perrottetii et précédemment (Tableau 1) à l'exception des D. velutina.

(ii) sols ferralitiques : D. argillacea, $D$. ciliaris, D. exilis, D. gayana, D. horizontalis, D. longiflora, D. patagiata.

(iii) sols ferrugineux tropicaux : $D$. aristulata, D. ciliaris, D. debilis, D. delicatula, D. gayana, D. horizontalis, D. longiflora, D. nuda, D. perrottetii.

(iv) sols hydromorphes : D. ciliaris, $D$. gayana, D. horizontalis, $D$. leptorhachis, D. longiflora, D. nuda, D. perrottetii, D. ternata.

(v) lithosols : D. argillacea, D. ciliaris, $D$. debilis, $D$. delicatula, $D$. diagonalis, $D$. exilis, D. gayana, D. longiflora, $D$. velutina.

(vi) sols peu évolués : D. gayana.

(vii) regosols : D. aristulata, D. ciliaris, $D$. horizontalis, D. longiflora.

(viii) vasières : $D$. ciliaris, $D$. exilis, $D$. gayana, D. horizontalis, $D$. leptorhachis, D. longiflora, D. velutina.

Le Tableau 1 montre plus clairement que les espèces affectionnent mieux les sols ferrugineux tropicaux qui sont prédominantes au Sénégal, les lithosols, les sols ferralitiques et hydromorphes. Moins fréquemment, elles poussent sur les vasières et les sols rouge-brun et bruns semi-arides à fertilité relativement faible. Les regosols et les sols peu évolués sont les moins affectionnés. Conformément aux travaux de Brink and Belays (2006), les résultats de l'étude attestent que $D$. exilis ne supporte pas les sols salins (halomorphes). Il en est de même pour ses apparentés sauvages. $D$. ciliaris et D. gayana se rencontrent sur presque l'ensemble des types de sols nommés sols peu évolués (pour $D$. ciliaris) et les regosols (pour D. gayana). Elles sont suivies de D. longiflora qui, à l'image de D. exilis dont elle semble être le plus proche parent, pousse sur des sols ferralitiques, des lithosols, des vasières. Cependant, D. longiflora a semble-t-il la particularité de supporter des sols plus variés tels que les sols ferrugineux tropicaux, les sols hydromorphes et les regosols. Quant à $D$. horizontalis, elle pousse sur les mêmes types de sols que $D$. ciliaris avec qui elle s'apparente morphologiquement à l'exception des lithosols. S'agissant de $D$. aristulata, considérée comme une espèce rare au Sénégal, elle pousse sur des sols ferrugineux tropicaux, des lithosols et regosols. Cette étude révèle notamment que certaines espèces semblent avoir une exigence vis-à-vis du type de sol. C'est le cas de D. debilis, $D$. diagonalis et $D$. patagiata, une espèce menacée de disparition (Diop et Mahamane, 2010), et $D$. ternata qui sont rencontrées respectivement sur des sols ferrugineux tropicaux, des lithosols, des sols ferralitiques et sur des sols hydromorphes.

\section{CONCLUSION}

Il découle des résultats que les espèces, prises dans leur ensemble, occupent l'ensemble du territoire sénégalais et donc des environnements très variés. Cependant au Sénégal, la plupart d'entre eux se trouvent dans le sud du pays où les conditions environnementales sont plus favorables. Les différents types de sols occupés par les espèces attestent de leur forte capacité d'adaptation. Les nombreuses propriétés intrinsèques des espèces sauvages comparées à celles de l'espèce cultivée laissent supposer que son pool génique primaire contient une source précieuse de diversité qui pourrait être utile pour son amélioration. Cette étude pourrait également servir de support de prise de décision pour assurer une gestion durable des ces ressources phytogénétiques. 


\section{REFERENCES}

Adoukonou-Sagbadja, H., Wagner, C., Dansi, A., Ahlemeyer, J., Dainou, O., Akpagana, K., Ordon, F. and Friedt, W. 2007. Genetic diversity and population differentiation of traditional fonio millet (Digitaria spp.) landraces from different agro-ecological zones of West Africa. Theoretical and Applied Genetics 115: 917-931. DOI: http:/ /dx.doi.org/10.1007/s00122-007-0618-x

Asfaw, A. 2006. The effects of obesity on doctor-diagnosed chronic diseases in Africa: Empirical results from Senegal and South Africa. Journal of Public Health Policy 27:250-64. DOI: http://dx.doi.org/ 10.1057/palgrave.jphp.3200089

Berhaut, J. 1967. Flore du Sénégal (2ème ed.). Sénégal : Editions Clairafrique.

Brink, M. and Belay, G. (Eds.). 2006. Plant resources of Tropical Africa 1. Cereals and pulses. PROTA Foundation, Wageningen, Netherlands / Backhuys Publishers, Leiden, Netherlands/CTA, Wageningen, Netherlands.

Centre de Suivi Ecologique (CSE). 2007. Caractérisation des systèmes de production agricole au Sénégal. Document de synthèse, Sénégal.

Diop, B.M., Gueye, M.C, Agbangba, C.E., Cisse, N., Deu, M., Diack, O., Fofana, A., Kane, N.A., Ndir, K.N., Ndoye, I., Ngom, A., Leclerc, C., Piquet, M., Vigouroux, Y., Zekraoui, L., Billot, C. and Barnaud, A. 2018. Fonio (Digitaria exilis (Kippist) Stapf): A socially embedded cereal for food and nutrition security in Senegal. Ethnobiology Letters 9(2):150-165. DOI: http://dx.doi.org/10.14237/ebl.9.2. 2018.1072

Diop, F.N. and Mahamane, A. 2010. Digitaria patagiata. The IUCN red list of threatened species 2010. DOI: http://dx.doi.org/ 10.2305/IUCN.UK.2010-3.RLTS.T185476 A8419120.en

Gueye, M., Kanfany, G., Fofana, A., Gueye, M., Noba, K. and Grove, J. 2015. Effect of Planting Date on Growth and Grain Yield of Fonio Millet (Digitaria exilis Stapf) in the Southeast of Senegal. International Journal of Biological and Chemical Sciences 9(2):581-592. DOI: http://dx.doi. org/10.4314/ijbcs.v9i2.1

Ministeİre de l'Agriculture. 1996. Seìneìgal : Rapport de Pays pour la Confeìrence Technique Internationale de la FAO sur les Ressources phytogeìneìtiques. Leipzig, Allemagne, 17-23 juin 1996.

Mbow, C. 2009. Potentiel et dynamique des stocks de carbone des savanes soudaniennes et soudano-guinéennes du Sénégal. Doctorat d'Etat. Université Cheikh Anta Diop de Dakar, Sénégal.

MEPN .1997. Senegal: Rapport National de Biodiversité. Ministère de l'Environnement et de la Protection de la Nature, Sénégal.

Paraiso, A., Sossou, A.C.G., Yegbemey, R.N. and Biaou, G. 2011. Analyse de la rentabilité de la Production du fonio (Digitaria exilis Stapf) dans la Commune de Boukombe au Bénin. Journal de la Recherche Scientifique de l'Université de Lomé (Togo), Série A 13(1):27-37.

Popkin, B.M., Adair, L.S. and Ng, S.W. 2012. Global nutrition transition and the pandemic of obesity in developing countries. Nutrition Reviews 70:3-21. DOI: http://dx.doi.org/ 10.1111/j.1753-4887.2011.00456.x

USAID. 2008. Chaine de valeur fonio Sénégal : Analyse et cadre stratégique d'initiatives pour la croissance de la filière. International Resources Group, Washington, D.C, US.

Vall, E., Andrieu, N., Beavogui, F. and Sogodogo, D. 2011. Les cultures de soudure comme stratégie de lutte contre l'insécurité alimentaire saisonnière en Afrique de l'Ouest : le cas du fonio (Dgitaria exilis Stapf). Cahiers Agriculture 20(4):294-300.

Vanden Berghen, C. 1991. Monocotylédones et Ptéridophytes. In: Berhaut, J. (Ed.). Flore illustrée du Sénégal (Vol. X). Dakar, Sénégal : Ministère du Développement Rural et de l'Hydraulique, Direction des Eaux et Forêts, Sénégal. 